\title{
Secondary Metabolites from Some Species of Albizzia: A Review
}

\author{
Mohammad Musarraf Hussain ${ }^{1}$, Faiza Tahia ${ }^{2}$ and Mohammad A. Rashid ${ }^{2}$ \\ ${ }^{1}$ Department of Pharmacy, Jagannath University, Dhaka-1100, Bangladesh \\ ${ }^{2}$ Department of Pharmaceutical Chemistry, University of Dhaka, Dhaka-1000, Bangladesh
}

Received: January 01, 2016; Accepted: January 10, 2016; Published (Web): February 17, 2016

\begin{abstract}
This review covers the secondary metabolites reported from five species of Albizzia. A total of fifty chemical constituents have been documented from A. anthelmintica, A. chinensis, A. julibrissin, A. lebbeck and A. myriophyla. Most of the phytoconstituents have been reported from A. julibrissin and A. lebbeck.
\end{abstract}

Key words: Secondary metabolites, Albizzia anthelmintica, Albizzia chinensis, Albizzia julibrissin, Albizzia lebbeck, Albizzia myriophyla

\section{Introduction}

The genus Albizzia belongs to the family Leguminosae consisting of 150 species that are widely distributed all over the world (Ghaly et al., 2010). A. anthelmintica is a throny medium canopied tree growing to $8 \mathrm{~m}$ height with smooth bark and open spinetipped twigs. A. chinensis (Bengali name: chakua, amlukia) is an evergreen tree with a flat spreading crown, 30m height with dark gray bark (Orwa et al., 2009). A. julibrissin is a small tree growing upto $5-16 \mathrm{~m}$ tall with araching branches, dark greenish grey bark for each plant and bipinnate leaves. A. lebbeck (Bengali name: Shirish or kalo koroi) is a tall tree that are 12-21 $\mathrm{m}$ high and grows all over Bangladesh. The flowers of this plant are useful in asthma and snake bite. The leaves are used in the treatment of blindness and syphilis. The bark is used in inflammation, toothache and leprosy. Having astringent property, the root is helpful in opthalmia and skin diseases (Hussain et al., 2008; Kirtikar and Basu, 1980; Rashid et al., 2003).
A. myriophyla is also a small tree having $4 \mathrm{~m}$ height with dark brown young shoots and bipinnate leaves (Orwa et al., 2009). Previous phytochemical studies on the genus of Albizzia revealed the occurrences of various natural products such as alkaloids (Dixit and Mitra, 1997), glycosides (Varshney, 1976), terpenoids, steroids and saponins (Pal et al., 1995), anthraquinines and phenolics (Deshpande and Shastri, 1977), triterpenoids, diterpenoids, lignans, and pyridineglycosides (Ghaly et al., 2010)

\section{Results}

Secondary metabolites: A total of 50 secondary metabolites have been reported from five species of Albizzia (A. anthelmintica, A. chinensis, A. julibrissin, A. lebbeck and A. myriophyla) as mentioned in table 1 and figure 1 . 
Table 1. Reported secondary metabolites from Albizzia species.

\begin{tabular}{|c|c|}
\hline Species & Secondary metabolites \\
\hline A. anthelmintica & $\begin{array}{l}\text { 3-O-[L-arabinopyranosyl }(1 \rightarrow 6)] \text {-2-acetamido-2-deoxy- } \beta \text {-D-Glucopyranosyl echinocystic acid (1) } \\
\text { (Carpani et al., 1989). }\end{array}$ \\
\hline A. chinensis & $\begin{array}{l}\text { Kaempferol 3-O- } \alpha \text {-L-rhamnopyranoside (2), Quercetin 3-O- } \alpha \text {-L-rhamnopyranoside (3), Luteolin (4), } \\
\text { Kaempferol (5), Quercetin (6) (Ghaly et al., 2010), } \alpha \text {-Amyrin (7), } \beta \text {-sitosterol (8), 7,3-dimethoxyluteolin } \\
\text { (9) (Rashid et al., 2014; Sharmin et al., 2014). }\end{array}$ \\
\hline A. julibrissin & 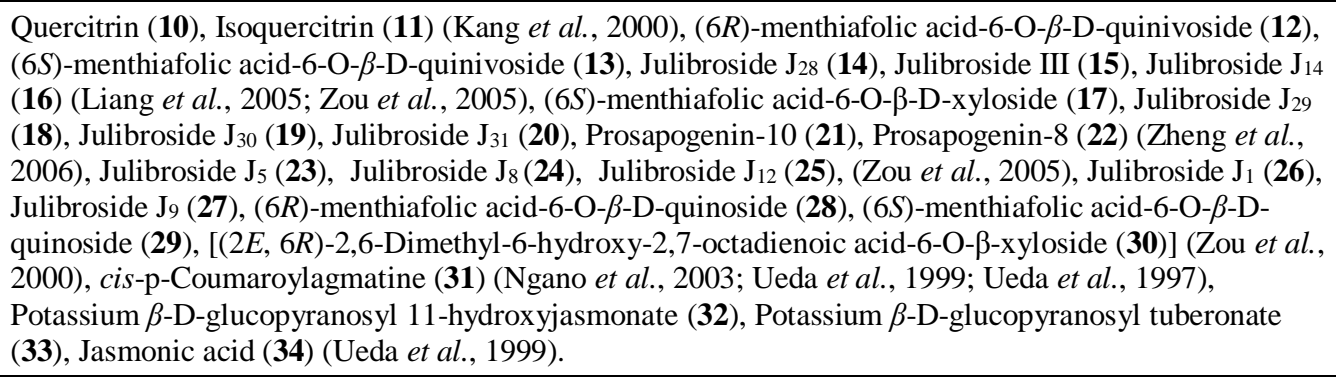 \\
\hline A. lebbeck & $\begin{array}{l}\text { Lupeol (35), Stigmasterol (36), 4-Hydroxy-3-methoxycinnamic acid (37), trans-p-coumaric acid (38) } \\
\text { (Hussin et al., 2008), 3',5-Dihydroxy-4',7-dimethoxy flavones (39), } N \text {-benzoyl-L-phenylalaninol (40) } \\
\text { (Rashid } \text { et al., 2003), Albigenin (41), Lebbekanin A (42), Lebbekanin E (43), Melacacidin (44), (-)-2,3- } \\
\text { cis-3,4-cis-3-O-methyl melacacidin (45), Melanoxetin (46), 3'-O-methylmelanoxetin (47) (Une et al., } \\
\text { 2001). }\end{array}$ \\
\hline A. myriophyla & Albizzioside A (48), Albizzioside (49), Albizzioside (50) (Ito et al., 1994). \\
\hline
\end{tabular}

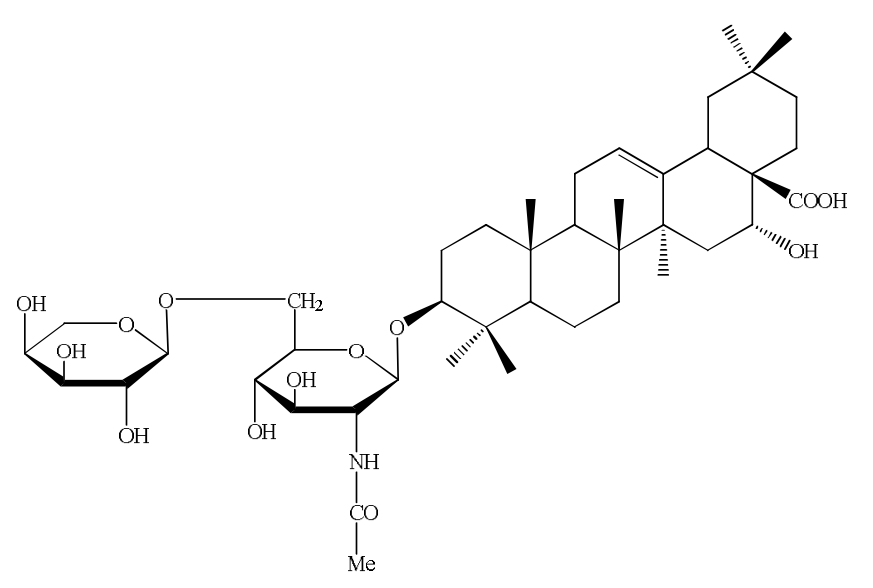<smiles>C[C@H]1CC[C@@]2(C)CC[C@]3(C)C(=CCC4[C@@]3(C)CC[C@]3(F)C(C)(C)C(O)CC[C@]43C)[C@]12C</smiles>

7<smiles>[R]c1cc(-c2oc3cc(O)cc(O)c3c(=O)c2[R])ccc1O</smiles>

2: $\mathrm{R}=\mathrm{O}$-alpha-L-rhamnopyranosyl, $\mathrm{R}_{1}=\mathrm{H}$

3: $\mathrm{R}=\mathrm{O}$-alpha-L-rhamnopyranosyl, $\mathrm{R}_{1}=\mathrm{OH}$

4: $\mathrm{R}=\mathrm{H}, \mathrm{R}_{1}=\mathrm{OH}$

5: $\mathrm{R}=\mathrm{OH}, \mathrm{R}_{1}=\mathrm{H}$

6: $\mathrm{R}=\mathrm{OH}, \mathrm{R}_{1}=\mathrm{OH}$

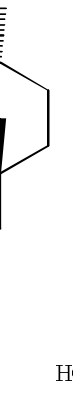<smiles>CCC(CCC(C)C1CCC2C3CC=C4C[C@@H](C)CCC4(C)C3CCC12C)C(C)C</smiles><smiles>O=c1cc(-c2ccc(OCCCCO)c(Cl)c2)oc2cc(O)cc(O)c12</smiles> 
<smiles>O=c1c(O)c(-c2ccc(O)c(O)c2)oc2c(O)c(O)ccc12</smiles>

10: $\mathrm{R}=$ Alpha-L-rhamnopyranose

11: $\mathrm{R}=$ Beta-D-glucopyranose

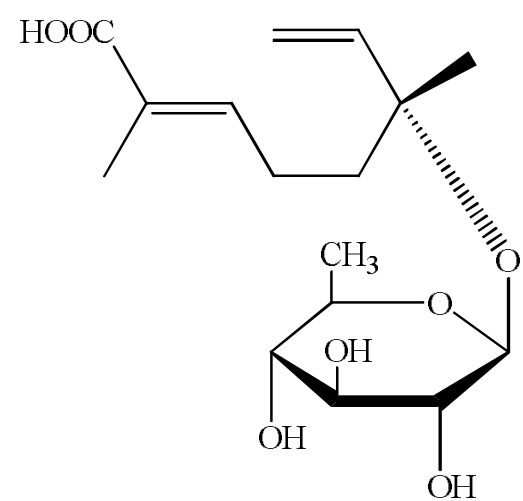

12

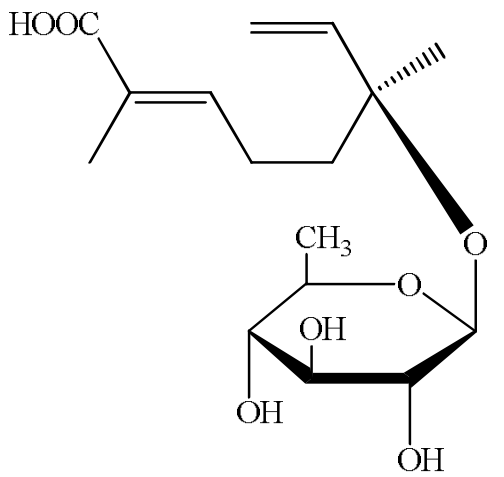

13

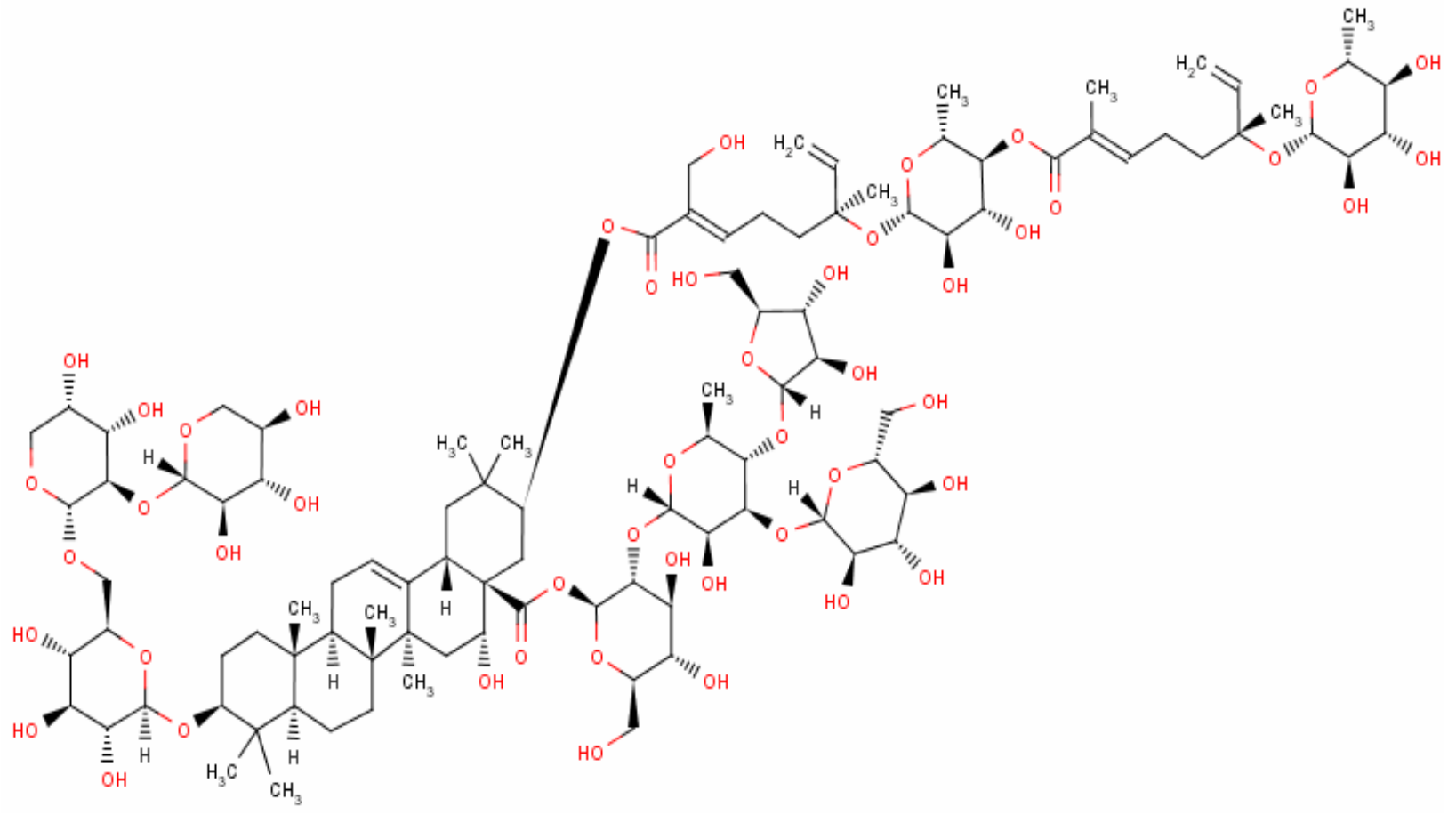




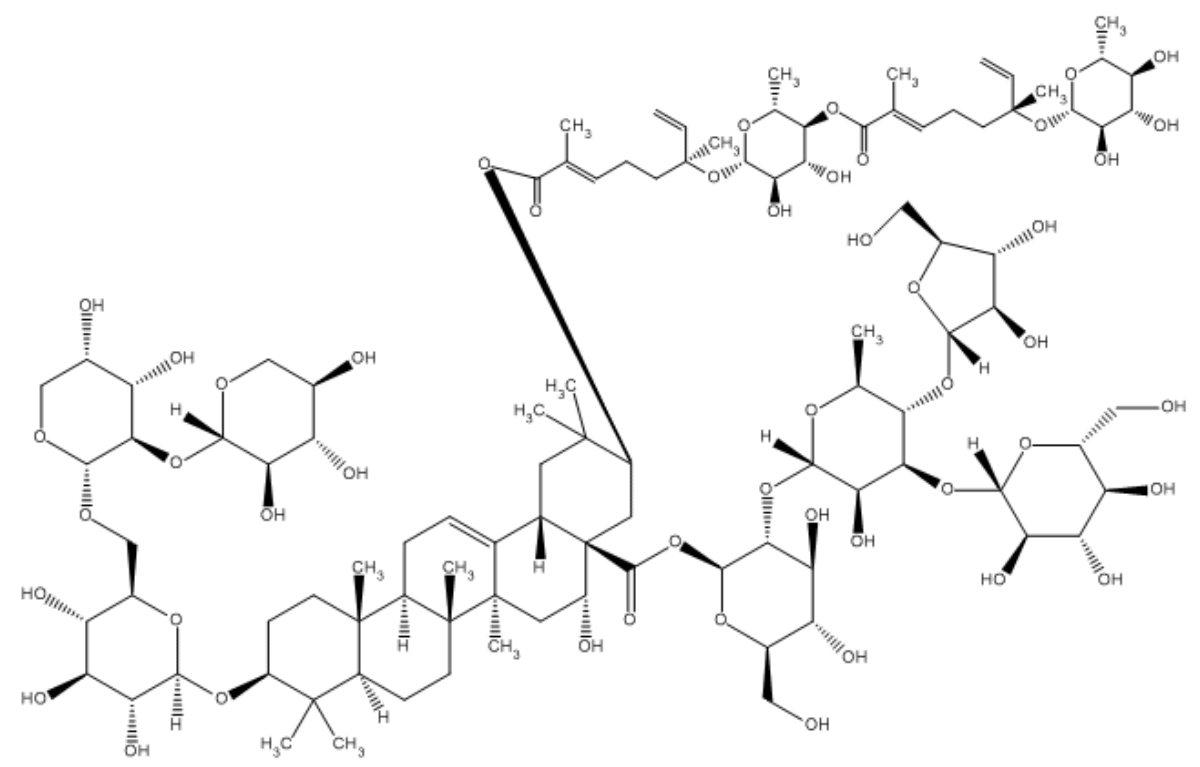

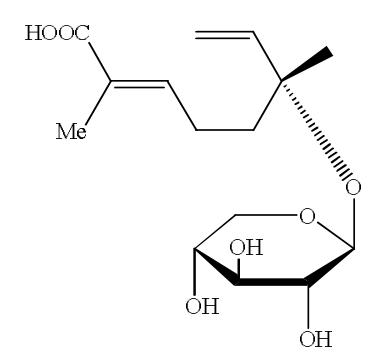

17

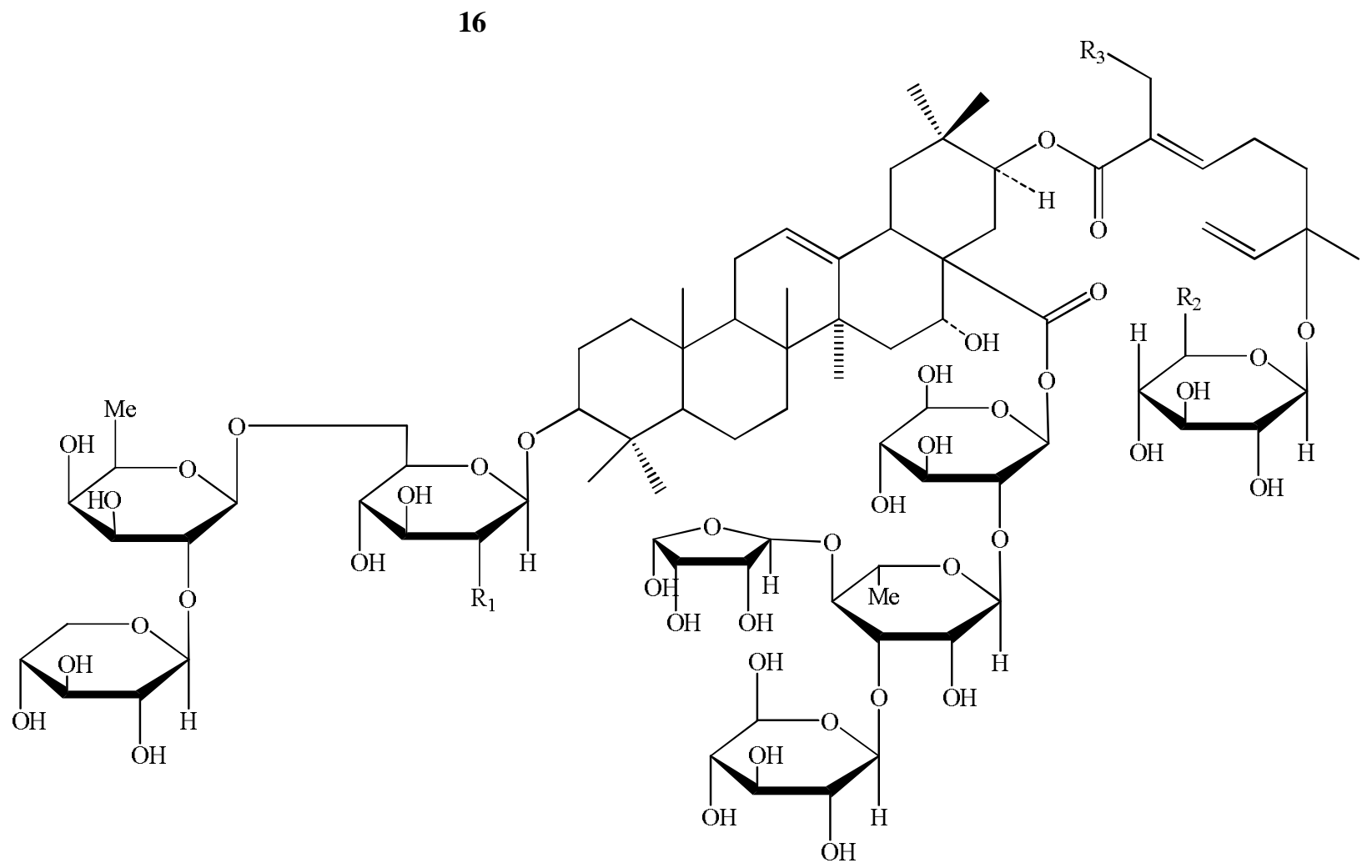

18: $\mathrm{R}_{1}=$ NHAc, $\mathrm{R}_{2}=\mathrm{Me}, \mathrm{R}_{3}=\mathrm{OH}$

19: $\mathrm{R}_{1}=\mathrm{NHAc}, \mathrm{R}_{2}=\mathrm{H}, \mathrm{R}_{3}=\mathrm{OH}$

20: $\mathrm{R}_{1}=\mathrm{O}$-glc, $\mathrm{R}_{2}=\mathrm{Me}, \mathrm{R}_{3}=\mathrm{OH}$

21: $\mathrm{R}_{1}=\mathrm{OH}, \mathrm{R}_{2}=\mathrm{Me}, \mathrm{R}_{3}=\mathrm{OH}$

22: $\mathrm{R}_{1}=\mathrm{O}$-glc, $\mathrm{R}_{2}=\mathrm{Me}, \mathrm{R}_{3}=\mathrm{H}$ 


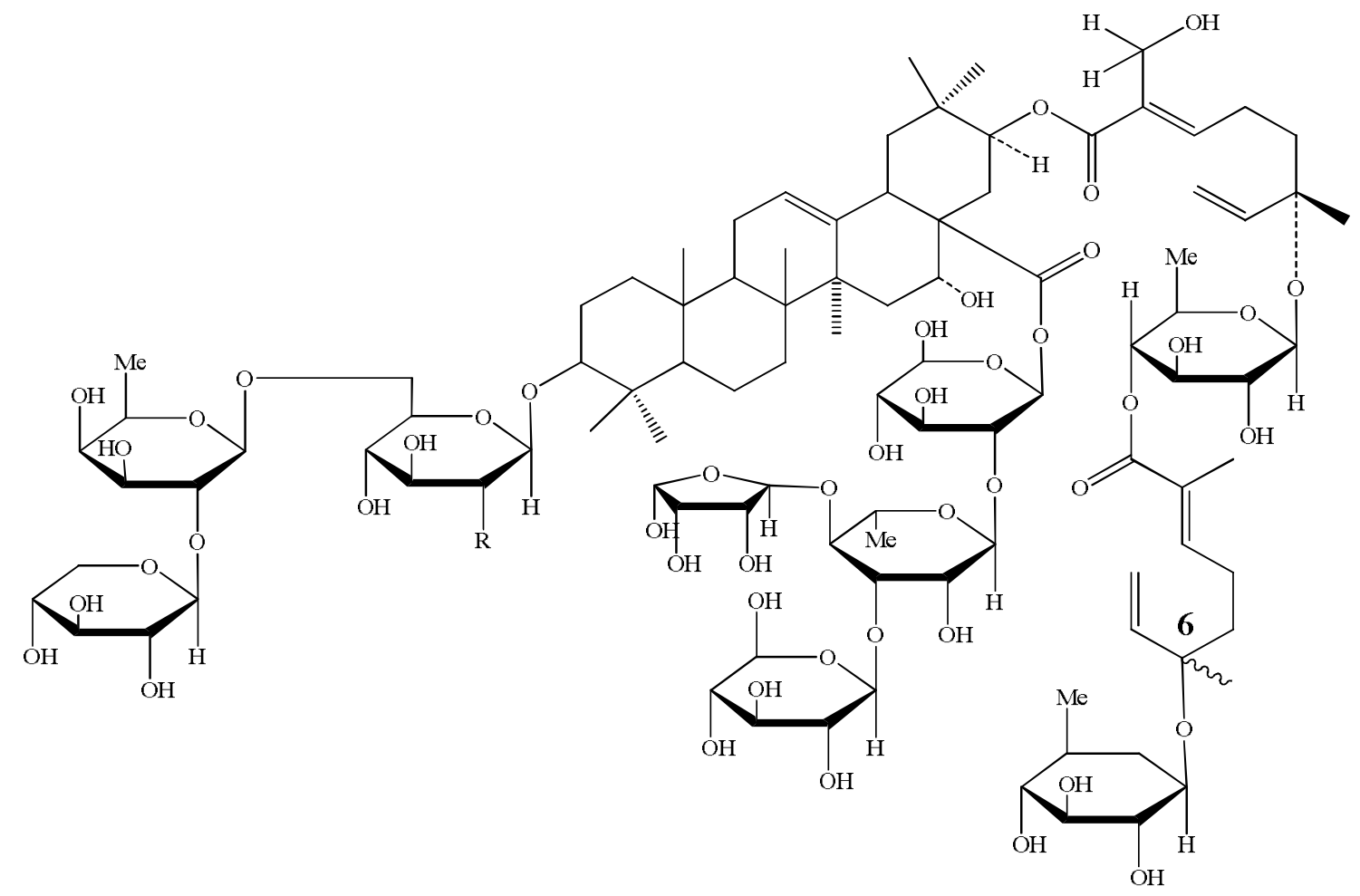

23: $\mathrm{R}=\mathrm{OH}, 6 \mathrm{R}$

24: $\mathrm{R}=\mathrm{OH}, 6 \mathrm{~S}$

25: $\mathrm{R}=\mathrm{NHAc}, 6 R$

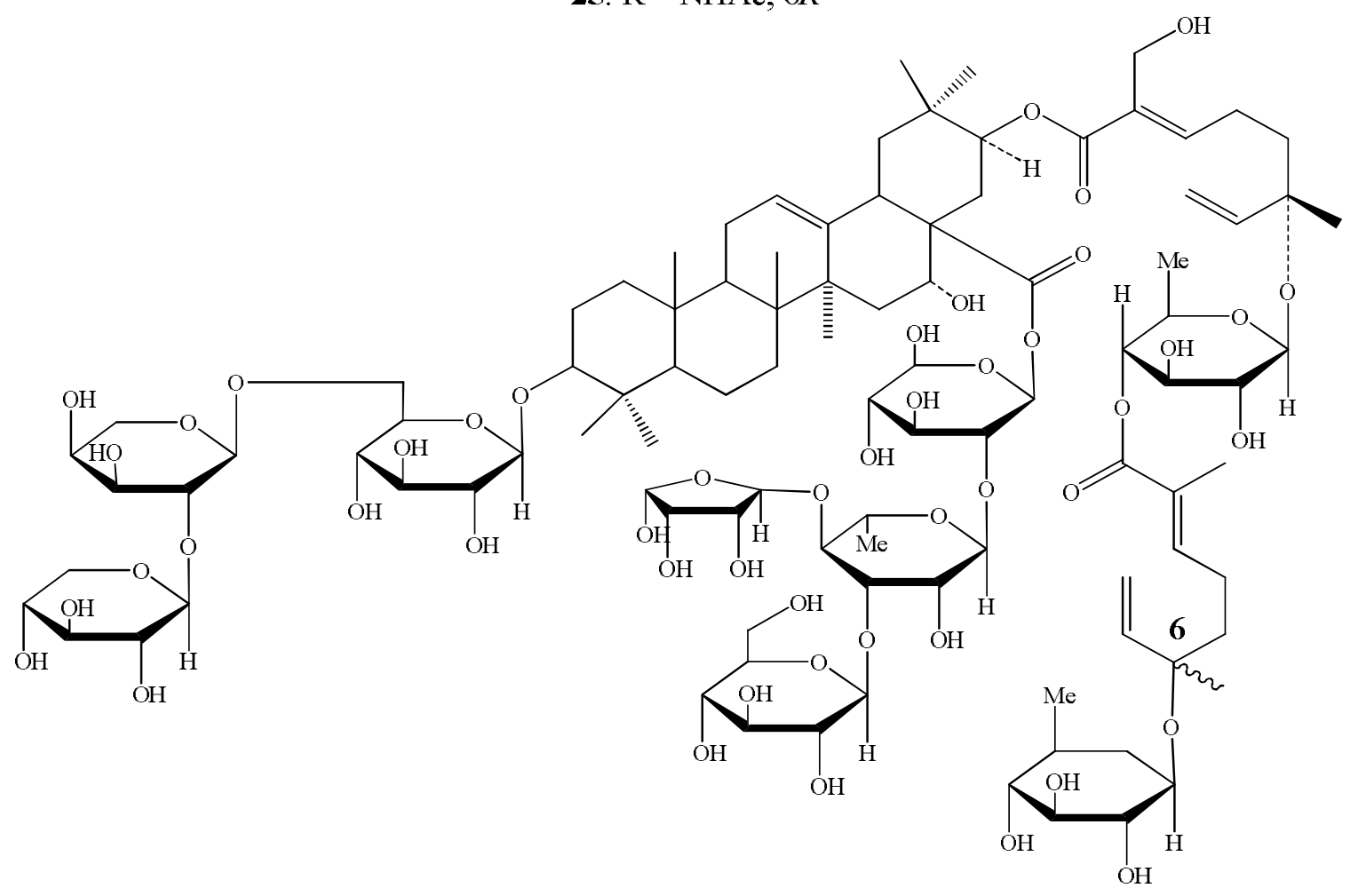

26: $\mathrm{C}-6=R$

27: $\mathrm{C}-6=S$ 

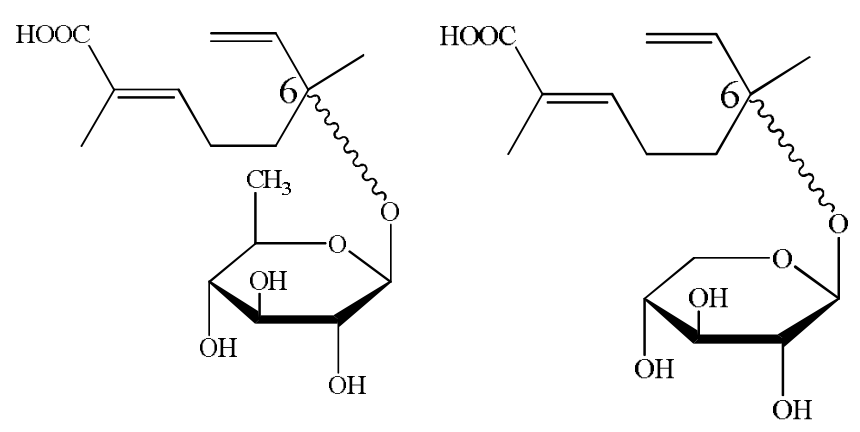

28: $\mathrm{C}-6=R$

29: $\mathrm{C}-6=S$

30: $\mathrm{C}-6=R$<smiles>NC(N)NCCCCNC(=O)/C=C\c1ccc(O)cc1</smiles><smiles>O=C(O)C[C@H]1CCC(=O)[C@@H]1C/C=C/CCOC(O)C(O)C(O)CO</smiles>

32

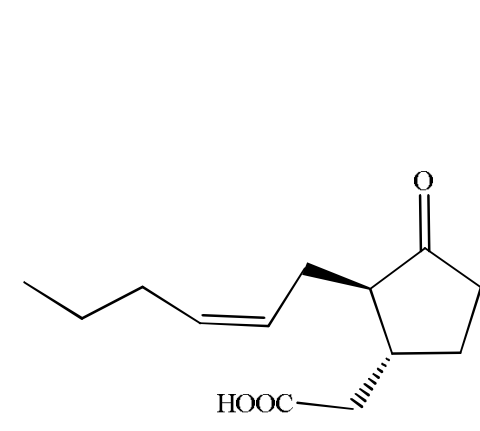

34

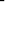<smiles>O=C(O)C[C@@H]1CCC(=O)[C@@H]1C/C=C/CCOC(O)[C@H](O)[C@H](O)C(O)CO</smiles>

33<smiles>[1H][C@@H]1CC(C)(C)C2CCC3(C)C(CCC4C5C(C(=C)C)CCC5(C)CCC43C)C2(C)C1</smiles>

35<smiles>CCC(/C=C/C(C)C1CCC2C3CC=C4CC(O)CCC4(C)C3CCC12C)C(C)C</smiles>

36<smiles>COc1cc(/C=C/C(=O)O)ccc1O</smiles>

37<smiles>O=C(O)/C=C/c1ccc(O)cc1</smiles>

38<smiles>COc1cc(O)c2c(=O)cc(-c3ccc(OC)c(O)c3)oc2c1</smiles>

39 
<smiles>O=CC(Cc1ccccc1)NC(=O)c1ccccc1</smiles>

40<smiles>CC1(C)C[C@H]2C3=CCC4[C@](C)(CCC5C(C)(C)[C@@H](O)CC[C@@]54C)[C@@]3(C)CC(O)[C@@]2(C(=O)O)C[C@@H]1O</smiles>

43<smiles>CC1(CC=O)CCCC2(C)[C@H]1CCC(C)(C)[C@H]2O</smiles>

41<smiles>CC1(C)CC[C@]2(C(=O)O)C(O)C[C@]3(C)C(=CCC4[C@@]5(C)CCC(O)CC5C(C)(C)C[C@]43C)C2C1</smiles>

42<smiles>COc1cc([C@@H]2CC[C@H]3[C@@H](c4cc(OC)c(O)c(OC)c4)CC[C@H]23)cc(O)c1O</smiles>

48: $\mathrm{R}=\mathrm{Glc}^{2}-\wedge \mathrm{pi}$ 49: $\mathrm{R}=\mathrm{II}$

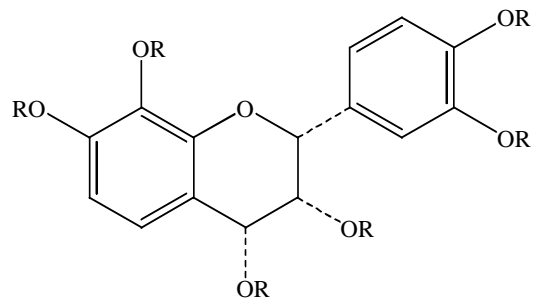

44: $\mathrm{R}=\mathrm{H}$<smiles>[R]c1cc(-c2oc3c(O)c(O)ccc3c(=O)c2O)ccc1O</smiles>

46: $\mathrm{R}=\mathrm{H}$

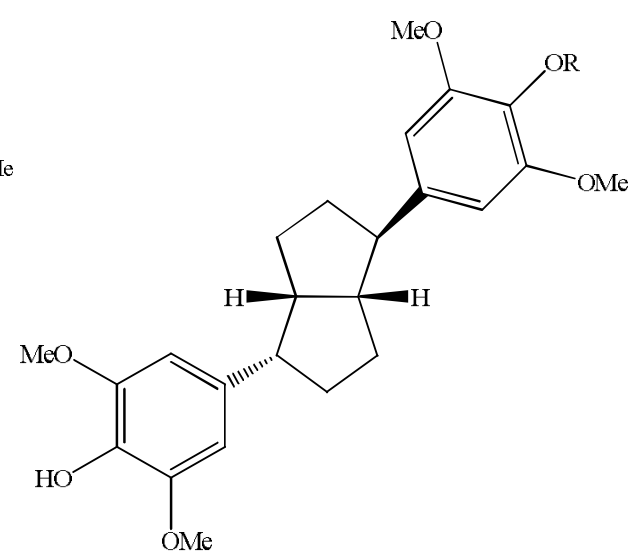

50: $\mathrm{R}=\mathrm{Glc}^{2}$-Api

Figure 1. Secondary metabolites from Albizzia species.

\section{Conclusion}

A total five species of Albizzia have been studied. Many diversified and structurally unique molecules have been reported from these plants. Our study revealed that Albizzia species can be a prominent source of secondary metabolites as well as drug candidates.

\section{References}

Carpani, G., Orsini, F., Sisti, M. and Verotta, L. 1989. Saponins from Albizzia anthelmintica. Phytochemistry 28, 863-866.

Deshpande, V.H. and Shastri, P.K. 1977. Phenolic of Albizzia lebbeck, A. amara, and A. Procera. Indian J. Chem. 15B, 201-204.

Dixit, A.K. and Misra, L.N. 1997. Macrocyclic budmunchiamine alkaloids from Albizzia lebbeck. Indian J. Nat. Pro. 60, 1036-1037. 
Ghaly, N.S., Melek, F.R. and Abdelwahed, N.A.M. 2010. Flavonoids from Albizzia chinensis of Egypt. Rev. Latinoamer. Quim. 38, 153-158.

Hussain, M.M., Rahman, M.S., Jabbar, A. and Rashid, M.A. 2008. Phytochemical and biological investigation of Albizzia lebbeck. Boletin Latinoamericano y del Caribe de Plantas Medicinales Y Aromat. 7, 273-278.

Ito, A., Kasai, R., Yamasaki, K., Duct, N.M. and Nham, N.T. 1994. Lignan glycosides from bark of Albizzia myriophylla. Phytochemistry 37, 1455-1458.

Kang, T.H., Jeong, S.J., Kim, N.Y., Higuchi, R. and Kim, Y.C. 2000. Sedative activity of two flavonol glycosides isolated from the flowers of Albizzia julibrissin durazz. $J$. Ethnopharmacol. 71, 321-323.

Kirtikar, K.R. and Basu, B.D. 1980. Indian Medicinal Plants, Published by Singh, B. and Singh, M.P., India $2^{\text {nd }}$ edition.

Liang, H., Tong, W.Y., Zhao, Y.Y., Cui, J.R. and Tu, G.Z. 2005. An antitumor compound Julibroside $\mathrm{J}_{28}$ from Albizzia julibrissin. Bioorg. Med. Chem. Lett. 15, 44934495.

Nagano, H., Kato, E., Yamamura, S. and Udea, M. 2003. Fluorescence studies on nyctinasty using fluorescence labeled cis-p-coumaroylagmatine, a leaf opening substance of Albizzia plants: existence of genus-specific receptor for leaf-movement factor. Tetrahedron Lett. 44, 2953-2956.

Orwa, C., Mutua, A., Kindt, R., Jamnadass, R. and Anthony, S. 2009. Agroforestree database: a tree reference and selection guide version 4

Pal, B.C., Achari, B., Yoshikawa, K. and Arihara, S. 1995. Saponins from Albizzia lebbeck. Phytochemistry 38, 1287-1291.

Rashid, M.A., Hauque, M.R., Sikder, M.A.A., Chowdhury, A.A., Rahman, M.S. and Hasan, C.M. 2014. Review on chemistry and bioactivities of secondary metabolites from some medicinal plants and microbes in Bangladesh. Bangladesh Pharm. J. 17, 63-79.
Rashid, R.B., Chowdhury, R., Jabbar, A., Hasan, C.M. and Rashid, M.A. 2003. Constituents of Albizzia lebbeck and antibacterial activity of an isolated flavones derivative. Saudi Pharm. J. 11, 52-56.

Sharmin, T., Islam, F., Kaiser, M.A., Al-Monsur, M.A., Sikder, M.A. and Rasid, M.A. 2014. Chemical and biological investigation of Albizzia chinensis (Osbeck.) Merr. J. Phy. Sci. 25, 29-39.

Ueda, M., Tashiro, C. and Yamamura, S. 1997. Cis-pCoumaroylagmatine, the genuine leaf-opening substance of a nyctinastic plant, Albizzia julibrissin durazz. Tetrahedron Lett. 38, 3253-3256.

Ueda, M. and Yamamura, S. 1999. Potassium $\beta$-Dglucopyranosyl 11-hydroxyjasmonate, a leaf-closing substance of Albizzia julibrissin durazz. Tetrahedron Lett. 40, 7823-7826.

Une, H.D., Pal, S.C., Kasture, V.S. and Kasture, S.B. 2001. Phytochemical constituents and pharmacological profile of Albizzia lebbeck. J. Nat. Reme. 1, 1-5.

Vaeshney, I.P. 1976. Glycosides and carbohydrates from the members of the family Leguminosae. Univ. Indore Res. J. Sci. 4, 13-22.

Zheng, L., Zheng, J., Zhao, Y., Wang, B., Wu, L. and Liang, H. 2006. Three anti-tumor saponins from Albizzia julibrissin. Boorg. Med. Chem. Lett. 16, 2765-2768.

Zou, K., Tong, W.Y., Liang, H., Cui, J.R., Tu, G.H., Zhao, Y.Y. and Zhang, R. 2005. Diastereomeric saponins from Albizzia julibrissin. Carbohydrate Res. 340, 1329-1334.

Zou, K., Zhao, Y., Tu, G., Cui, J., Jia, Z. and Zhang, R. 2000. Two diastereomeric saponins with cytotoxic activity from Albizzia julibrissin. Carbohydrate Res. 324, 182188. 